\title{
COMPUTATION OF INFRARED HYDROGEN LINES
}

\author{
MATS CARLSSON \\ Institute of Theoretical Astrophysics, P.O. Box 1029, Blindern, N-0315 Oslo 3, Norway \\ and \\ ROBERT J. RUTTEN \\ Sterrekundig Instituut, Postbus $80000, N L-3508$ TA, Utrecht, The Netherlands
}

\begin{abstract}
We compare infrared hydrogen lines observed with ATMOS with computations for two models of the solar atmosphere, one without and one with a chromosphere. The weaker H I lines are formed in the photosphere. Proper evaluation of Stark broadening is required to reproduce their profiles; the heavy ion contribution is most important. The cores of the stronger lines are sensitive to the structure of the chromosphere, but detailed NLTE modeling is needed for diagnostic applications.
\end{abstract}

Key words: atomic processes - hydrogen - infrared: stars - line: formation - line: profiles - Sun: atmosphere

\section{Introduction}

In an early review of the infrared solar spectrum, de Jager (1964) summarized his pioneering H I Paschen and Brackett studies (de Jager and Neven, 1950; de Jager et al., 1956). He suggested that it would be worthwhile to compare the infrared hydrogen lines with modern broadening theory and noted that Pfund $\alpha$ and the next $\alpha$ line (6-7) should be observable from $20 \mathrm{~km}$ or higher altitude. A quarter century later, the ATMOS space interferometer (Farmer and Norton, 1989) has finally yielded data which allow us to follow his suggestion. We do that here, using an ATMOS atlas kindly forwarded by R. Kurucz. More detail is given in Carlsson and Rutten (1992) and elsewhere in these proceedings (Rutten and Carlsson, 1993).

\section{Collisional Broadening}

Figure 1 shows relative $\mathrm{H}$ I broadening contributions against $\lg \tau_{500}$ for the MACKKL quiet-Sun model atmosphere (Maltby et al., 1986). In the deepest photosphere $\left(\lg \tau_{500}>0\right)$, the broadening is dominated by Stark broadening due to protons. This contribution falls off quickly with height and then peaks again in the low chromosphere, above the temperature minimum, which is at $\lg \tau_{500}=-4$. Electron broadening shows a similar pattern, with an outward rise from increasing metal ionization. Throughout the photosphere, where the damping wings of the H I absorption lines are formed, Stark broadening by metal ions outweighs all other contributions except for the van der Waals broadening of Brackett $\alpha$ (top left panel).

\section{Line Profiles}

Figure 2 shows ATMOS data and computed $\mathrm{H}$ I line profiles, in the form of radiation temperatures to cancel variations in Planck function sensitity with wavelength. All panels have the same temperature scale to facilitate comparison; similarly, each panel spans $\pm 100 \mathrm{~km} \mathrm{~s}^{-1}$ in Dopplershift from line center. 



Fig. 1. Broadening contributions for infrared $\mathrm{HI}$ lines against continuum optical depth. Solid: Stark broadening by ions. Dot-dashed:Stark broadening by protons. Dashed: Stark broadening by electrons. Dotted: van der Waals broadening. 

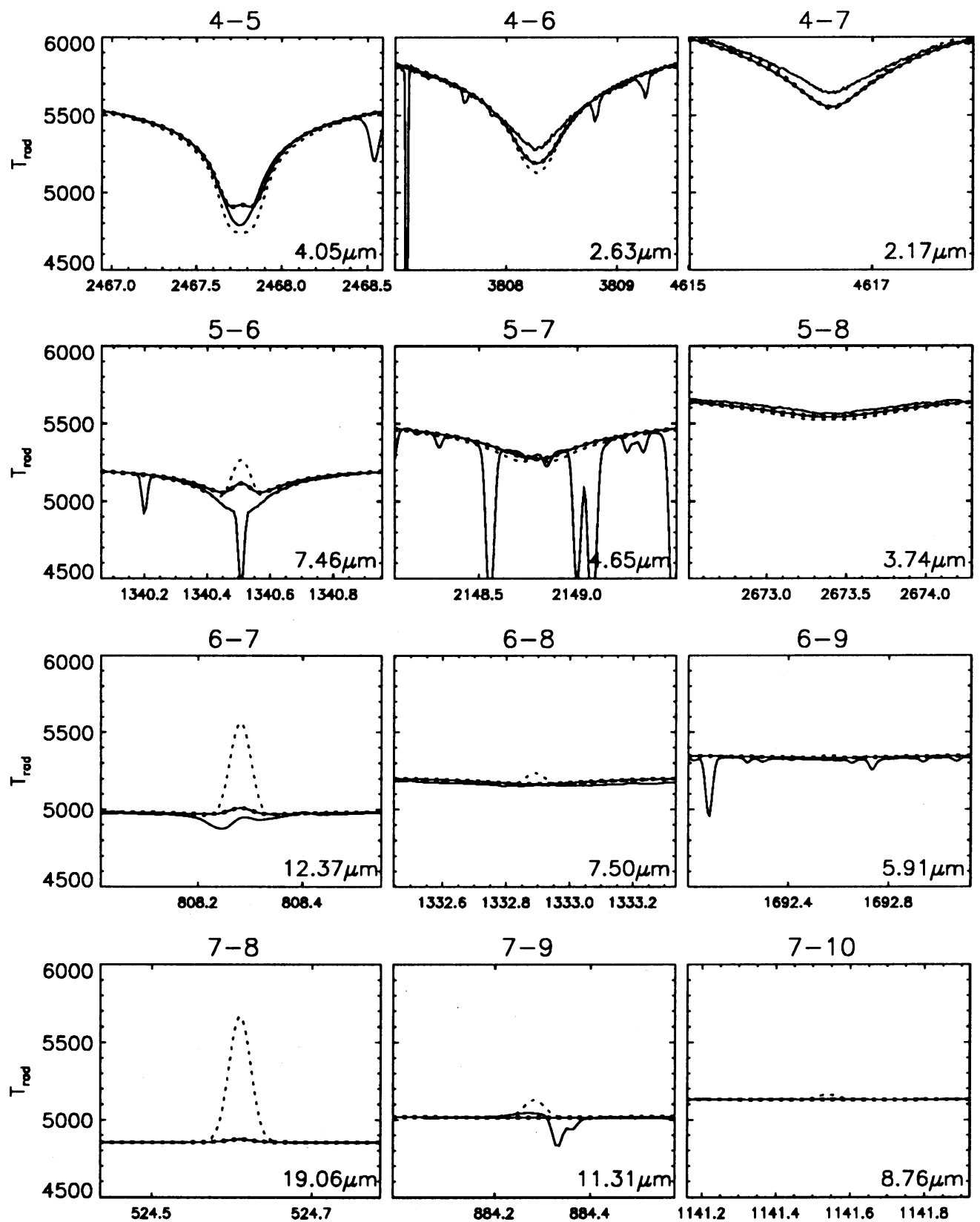

Fig. 2. Comparison of observed spectra from ATMOS (solid) with H I line profiles computed from MACKKL (dashed) and T5780 (solid+dashed). All profiles are normalized to the continuum intensity computed from the $\mathrm{T} 5780$ model. Ordinates: radiation temperature in K. Abscissae: wavenumber in $\mathrm{cm}^{-1}$. The line-center wavelength in $\mu \mathrm{m}$ is given in each panel. 

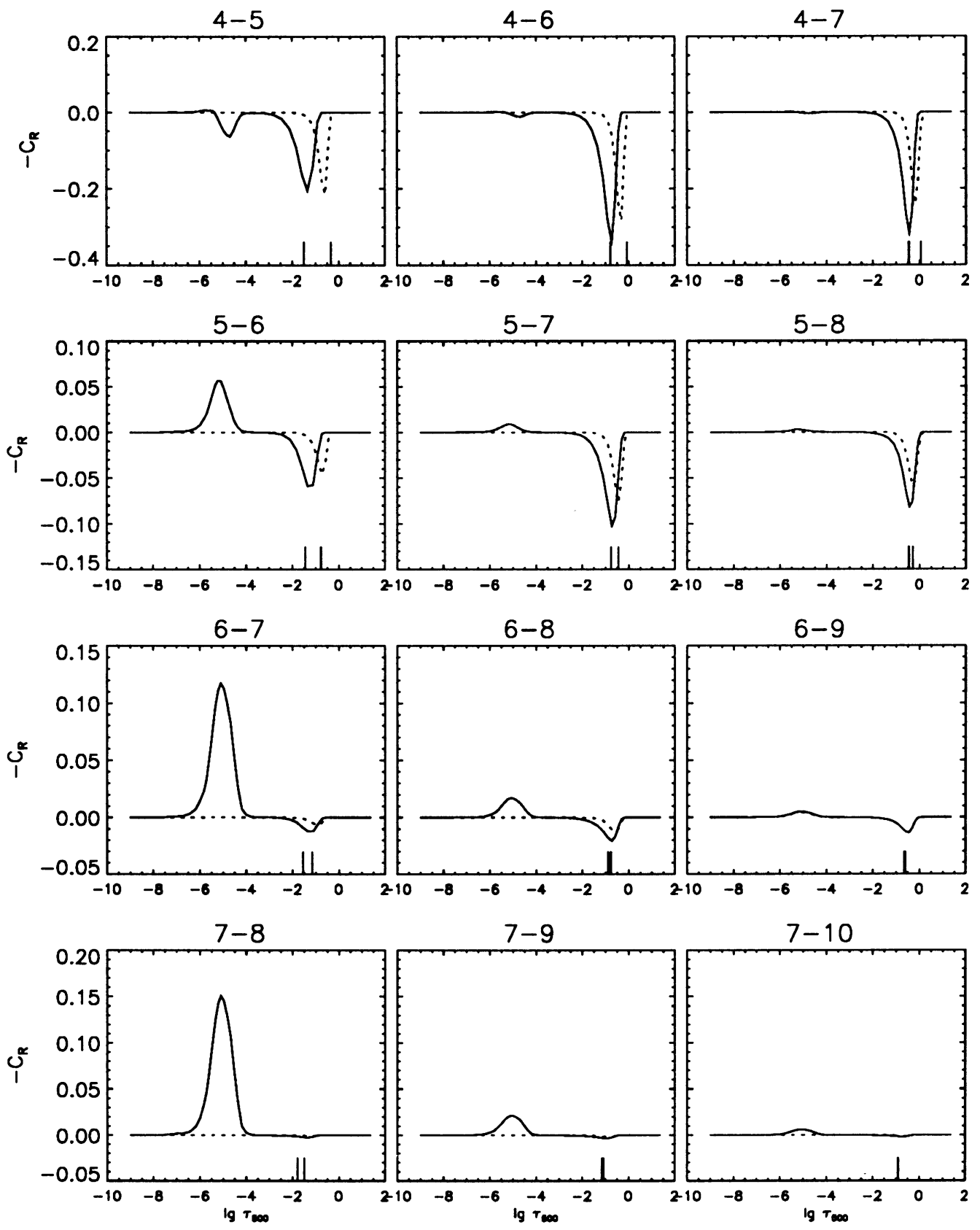

Fig. 3. H I relative-emission contribution functions for MACKKL against $\lg \tau_{500}$. Solid: at line center. Dashed: for $\Delta \lambda$ corresponding to the half-maximum value of the emergent line depression. The ticks on the abscissae mark the $\tau_{\nu}=1$ locations at line center and in the adjacent continuum. 
The dashed profiles are for the MACKKL model, the solid-plus-dashed for the T5780 model. The latter is a radiative-equilibrium model without chromosphere from Uppsala (Edvardsson et al., 1993). The wide wings of the photospheric profiles are reproduced very well. In the line cores, there are noticeable differences between computations and observations, and also between the two models.

The extinction coefficient of the $\alpha$ lines increases with the lower-level statistical weight, downward along the lefthand column of Fig. 2. Nevertheless, the lines weaken in equivalent width due to the steep increase of the $\mathrm{H}^{-}$free-free continuum opacity with wavelength (Figure 1 of Carlsson and Rutten, 1992). The 4-5 line is shallowest for the T5780 computation, in which the core is filled in by NLTE departure divergence. The MACKKL computation has a deeper core, slightly deeper than the observed profile. The other $\alpha$ lines show a reversed pattern. The T5780 computations have weak emission reversals due to NLTE effects, while the MACKKL produces additional emission from its chromosphere. LTE computations with the MACKKL give peaks of about half the NLTE values (Figure 3 of Carlsson and Rutten, 1992). The absorption spike in the 5-6 line is due to gas within the ATMOS interferometer. In the observed 6-7 spectrum, H I seems to cause the slight upward hump, not the dip at left. The 7-8 line was not observed by ATMOS. Its LTE MACKKL peak height is half the NLTE value shown here. The corresponding LTE line-center intensity is $I_{0} / I_{c}=1.07$, which corresponds better to the observation of Boreiko and Clark (1986) than the NLTE value.

The line and the continuum extinction decrease both to the right along the rows in Figure 2. Both models produce slightly too deep 4-6 and 4-7 lines. The 5-7 and 5-8 lines are reproduced very well. The higher $\beta$ and $\gamma$ lines (6-8 to 7-10) are better modeled by the T5780 atmosphere than by the MACKKL model. The latter again predicts too much line-center emission.

\section{Contribution Functions}

Figure 3 shows relative-emission contribution functions for MACKKL. These are line-depression contribution functions as defined by Magain (1986), plotted on reversed scales to let positive contributions correspond to line emission above the continuum. The $\tau_{\nu}=1$ locations for line center and the continuum are given by vertical tick marks on the abscissae. Their convergence with increasing wavelength (downward along the columns) illustrates that the continuum gains more opacity than the lines.

The $\tau_{\nu}=1$ locations all lie within the photosphere. The strongest, highestformed line (7-8) reaches unit opacity below $\lg \tau_{500}=-2$. Figure 1 of Carlsson and Rutten (1992) shows that even the H I 18-19 line reaches $\tau_{\nu}=1$ only at the temperature minimum.

Nevertheless, the $\mathrm{HI}$ lines are sensitive to the chromosphere far above these $\tau_{\nu}=$ 1 locations. The H I Rydberg populations have a dip at the temperature minimum (Figure 1 of Rutten and Carlsson, 1993). The line-to-continuum extinction ratio increases rapidly with height above the temperature minimum (Figure 1 of Carlsson and Rutten, 1992). Plots of the variation of $\tau_{\nu}$ with $\tau_{500}$ show plateaus at the temperature minimum. The lines therefore reach substantial optical depth above 
the temperature minimum, even though their $\tau_{\nu}=1$ location is in the photosphere. It causes the appreciable chromospheric relative-emission contributions in Figure 3.

\section{Discussion}

Various improvements in modeling infrared H I lines are desirable. First, Stark broadening should be computed with better accuracy. Ion densities should be quantified per species; the Holtsmark distribution should be used; and Stark shifts should be accounted for in detail. Unfortunately, atomic physics theory does not provide definitive recipes yet; the deeper-formed $\mathrm{HI}$ lines may help in formulating these.

Second, the presence of NLTE population departure divergences in the upper photosphere requires more investigation (cf. Rutten and Carlsson, 1993). Overionization in the Balmer continuum is probably the main driving process, but experimentation should bear that out. A "multi-MULTI" sensitivity analysis ( $c f$. Carlsson et al. 1992a, 1992b) may identify the role of various population and depopulation processes.

Third, the cores of the $\mathrm{HI} \alpha$ lines are sensitive to the structure of the chromosphere. Zirker (1985) showed that their off-limb emission strengths, as observed by Brault and Noyes (1983), fit characteristic prominence properties; their disk-center and center-to-limb profile shapes may constrain empirical models of the solar atmosphere. This sensitivity makes the infrared $\mathrm{H} I$ lines interesting, and makes detailed NLTE modeling of their formation worthwhile. The differences between profiles with and without a chromosphere in Figure 2 and between NLTE and LTE peak heights in Figure 3 of Carlsson and Rutten (1992) represent a first step. Other comparisons are discussed by Avrett (1993) in this volume.

\section{References}

Avrett, E. H.: 1993, in these proceedings.

Boreiko, R. T. and Clark, T. A.: 1986, Astron. Astrophys. 157, 353.

Brault, J. and Noyes, R.: 1983, Astrophys. J. 269, L61.

Carlsson, M. and Rutten, R. J.: 1992, Astron. Astrophys. 259, L53.

Carlsson, M., Rutten, R. J., and Shchukina, N. G.: 1992a, Astron. A strophys. $253,567$.

Carlsson, M., Rutten, R. J., and Shchukina, N. G.: 1992b, in M. S. Giampapa and J. A. Bookbinder (Eds.), Cool Stars, Stellar Systems, and the Sun, Proc. Seventh Cambridge Workshop, ASP Conf. Series, 26, p. 518.

de Jager, C.: 1964, in M. Migeotte (Ed.), Les Spectres Infrarouges des Astres, Coll. Int. Liège, Mém. $8^{\circ}$ Soc. Roy. Sci., Liège, p. 151.

de Jager, C., Migeotte, M., and Neven, L.: 1956, Ann d'Astrophys. $19,9$.

de Jager, C. and Neven, L.: 1950, Proc. Kon. Ned. Akad. Wetensch. 53, 1578.

Edvardsson, B., Gustafsson, B., Lambert, D. L., Nissen, P. E., Tomkin, J., and Andersen, J.: 1993, Astron. Astrophys., in press.

Farmer, C. B. and Norton, R. H.: 1989, A High-Resolution Atlas of the Infrared Spectrum of the Sun and the Earth Atmosphere from Space, NASA Ref. Publ. 1224, Vol. 1.

Magain, P.: 1986, Astron. Astrophys. 163, 135.

Maltby, P., Avrett, E. H., Carlsson, M., Kjeldseth-Moe, O., Kurucz, R. L., and Loeser, R.: 1986, Astrophys. J. 306, 284.

Rutten, R. J. and Carlsson, M.: 1993, in these proceedings.

Zirker, J. B.: 1985, Solar Phys. 102, 33 